\title{
Study of Ultrasonic Velocity Parameters of Multi-Charged Electrolytes (Potassium Ferri and Ferro Cyanide) in Aqueous Dextrose Solutions at 298.15 K
}

\author{
SUJIT KUMAR DEHURY*, M. TALUKDAR and U. N. DASH \\ Department of Chemistry, Institute of Technical Education and Research (ITER) Siksha 'O' \\ Anusandhan University, Bhubaneswar, 751030, India \\ sujitam@rediffmail.com
}

Received 29 March 2013 / Accepted 30 April 2013

\begin{abstract}
The experimental ultrasonic velocity values have been utilized to compute specific acoustic impedance $(Z)$, molar sound velocity $(R)$, molar compressibility $(\mathrm{W})$, free volume $\left(V_{f}\right)$, isentropic compressibility $\left(K_{\mathrm{s}}\right)$, internal pressure $\left(\pi_{i}\right)$ and relative association $\left(R_{A}\right)$ for the solutions of potassium ferri and ferrocyanide in aqueous dextrose solutions at $298.15 \mathrm{~K}$. The results have been discussed considering all the parameters in terms of solute-solvent and solute-solute interactions and various structural effects.
\end{abstract}

Keywords: Ultrasonic velocity, Dextrose, Density, Potassium ferri and ferrocyanide

\section{Introduction}

Dextrose is a water soluble sugar and acts as a source of instant energy to human system. It is available in the world market in different brand names. Pure aqueous dextrose solution is generally used in saline, ORS-Electrokind packets and used to conduct the blood sugar test. Dextrose is a big deal for many researchers to study the ion solvent interactions in its mixed solvents because of its extensive pharmaceutical, biomedical and industrial applications. The present investigation has been carried out to study the interactions of multi-charged electrolytes (potassium ferricyanide and ferrocyanide) with aqueous dextrose (5, 10 and $15 \mathrm{wt} \%$ ) solutions at $298.15 \mathrm{~K}$ and at atmospheric pressure by means of ultrasonic velocity measurements. In continuation of our earlier work in dextrose solution ${ }^{1}$, we aims at studying various important ultrasonic parameters like acoustic impedance $(Z)$, molar sound velocity $(R)$, molar compressibility $(W)$, free volume $\left(V_{f}\right)$, isentropic compressibility $\left(K_{s}\right)$ and internal pressure $\left(\pi_{i}\right)$ for both the electrolytes. The results have been analyzed considering the structural effects of the solute and solvent in solutions and in the light of ion-ion and ion-solvent interactions.

\section{Experimental}

All chemicals used were of G.R., B.D.H. or AnalaR grades. Conductivity water (Sp. cond. $\left.\sim 10^{-6} \mathrm{~S} \mathrm{~cm}^{-1}\right)$ was used for preparing water + dextrose $(5,10$ and $15 \%$, (w/w) $)$ mixtures. 
The dextrose content in the mixed solvents was accurate to within $\pm 0.01 \%$. The electrolyte solutions were prepared on the molal basis and conversion of molality to molarity was done by using the standard expression ${ }^{2}$ using the density values of the solutions determined at $298.15 \mathrm{~K}$. Solutions were kept for $2 \mathrm{~h}$ in a water thermostat maintained at the required temperature accurate to within $\pm 0.05 \mathrm{~K}$ before use for density measurements. Density measurements were done using a specific gravity bottle of $25 \mathrm{~mL}$ capacity. At least five observations were taken and differences in any two readings did not exceed $\pm 0.02 \%$. Ultrasonic velocity measurements on the solutions of both the electrolytes were made by using an Ultrasonic Interferometer (Mittal Enterprises, New Delhi, India) at a frequency of $2 \mathrm{MHz}$ at $298.15 \mathrm{~K}$ only. The accuracy of the ultrasonic velocity measurement is within $\pm 0.5 \mathrm{~m} / \mathrm{s}$. The electrolyte content of the solutions varied over a concentration range of $1.0 \times 10^{-2}$ to $1.0 \times 10^{-1} \mathrm{~mol} \mathrm{dm}{ }^{-3}$ for all these measurements.

\section{Theory}

From the ultrasonic velocity and density data, the values of isentropic compressibility, $K_{\mathrm{s}}$, apparent isentropic compressibility, $K_{s \phi}$, acoustic impedance (Z) and relative association $\left(R_{A}\right)$ have been calculated by using equations (1)-(4), respectively ${ }^{3-6}$.

$$
K_{s}=\left(1 / U^{2} d\right)
$$

where, $\mathrm{U}=$ ultrasonic velocity of the solution

$\mathrm{d}=$ density of the solution

$$
\begin{aligned}
K_{s, \phi} & =1000 K_{s} c^{-1}-K^{0}{ }_{s} d_{0}^{-1}\left(1000 c^{-1} d-M\right) \\
Z & =U d \\
R_{A} & =\left(\rho / \rho_{\mathrm{o}}\right) \mathrm{x}(\mathrm{Uo} / \mathrm{U})^{1 / 3}
\end{aligned}
$$

The molar sound velocity $(R)$, free volume $\left(V_{f}\right)$, molar compressibility $(W)$ and the increase in internal pressure $\left(\pi_{i}\right)$ due to electrostatic field of the ion have also been calculated by using the following equations, respectively ${ }^{7-11}$,

$$
\begin{aligned}
R & =\bar{M} d^{-1} U^{1 / 3} \\
V_{f} & =V_{m}-b \\
W & =K_{s}^{-1 / 7} \bar{M} d^{-1}
\end{aligned}
$$

and

$$
\text { Where, } \quad \begin{aligned}
\pi_{i} & =\left(K_{s}^{0}-K_{s}\right) \\
\bar{M} & =\frac{\sum n_{i} m_{i}}{\sum n_{i}}, \quad V_{m}=\frac{\bar{M}}{d} \\
b & =\frac{\bar{M}}{d}\left[1-\left(\frac{R T}{\bar{M} U^{2}}\right)\left\{\left(1+\frac{\bar{M} U^{2}}{3 R T}\right)^{1 / 2}-1\right\}\right]
\end{aligned}
$$

\section{Results and Discussion}

The measured value of ultrasonic velocity $(U)$ has been presented in Table 1. As observed, ultrasonic velocity $(U)$ values tend to increase with the increase of dextrose content in water 
and of concentration of potassium ferri and ferrocyanide in various solvent systems. This suggests that disruption of water structure is enhanced with the addition of dextrose and the solutes. This result is in agreement with the earlier studies ${ }^{3-6}$ made in various mixed solvents.

Table 1. Ultrasonic velocity $U(\mathrm{~m} / \mathrm{s})$ and density $\rho\left(\mathrm{kg} / \mathrm{m}^{3}\right)$ of potassium ferri and ferrocyanide in water+ dextrose mixtures at $298.15 \mathrm{~K}$

\begin{tabular}{ccccccc}
\hline $\mathrm{C}$ & $5.0 \mathrm{wt} \%$ Dextrose & \multicolumn{2}{c}{$10.0 \mathrm{wt} \%$ Dextrose } & \multicolumn{1}{c}{$15.0 \mathrm{wt} \%$ Dextrose } \\
\cline { 2 - 7 } $\mathrm{mol} / \mathrm{dm}^{3}$ & $U$ & $\rho$ & $U$ & $\rho$ & $U$ & $\rho$ \\
\hline \multicolumn{7}{c}{ Potassium Ferricyanide } \\
0.01 & 1518.8 & 1017.2 & 1532.0 & 1036.8 & 1544.4 & 1057.3 \\
0.02 & 1520.4 & 1019.7 & 1537.6 & 1039.4 & 1552.0 & 1059.8 \\
0.04 & 1530.0 & 1022.8 & 1546.0 & 1042.9 & 1556.0 & 1062.6 \\
0.06 & 1536.8 & 1026.0 & 1552.0 & 1045.4 & 1560.0 & 1064.9 \\
0.08 & 1538.4 & 1029.7 & 1553.2 & 1049.4 & 1564.0 & 1069.1 \\
0.1 & 1549.6 & 1033.1 & 1559.2 & 1052.7 & 1580.0 & 1072.1 \\
0.01 & 1524.0 & 1019.8 & 1538.8 & 1039.1 & 1545.6 & 1060.8 \\
0.02 & 1528.0 & 1023.8 & 1549.2 & 1042.8 & 1556.8 & 1063.5 \\
0.04 & 1532.0 & 1027.1 & 1552.4 & 1046.5 & 1570.0 & 1067.3 \\
0.06 & 1540.0 & 1031.7 & 1554.0 & 1050.4 & 1575.2 & 1070.6 \\
0.08 & 1542.8 & 1037.0 & 1560.0 & 1055.8 & 1588.0 & 1076.1 \\
0.1 & 1552.0 & 1040.6 & 1576.0 & 1059.5 & 1602.4 & 1079.8 \\
\hline
\end{tabular}

The specific acoustic impedance $(Z)$ values have been calculated in aqueous solutions of 5,10 and $15 \mathrm{wt} \%$ dextrose at $298.15 \mathrm{~K}$. It is found that the values of $Z$ increase with increase in dextrose content in water as well as with increase in solute concentration in all the mixed solvents. This is in agreement with the theoretical requirements as both $U$ and density ( $\rho$ ) increase with increase in concentration of solute. Typical plots of $Z$ versus $c$ of the solutions of potassium ferri and ferrocyanide are shown in Figure 1 for different wt $\%$ of dextrose solution.

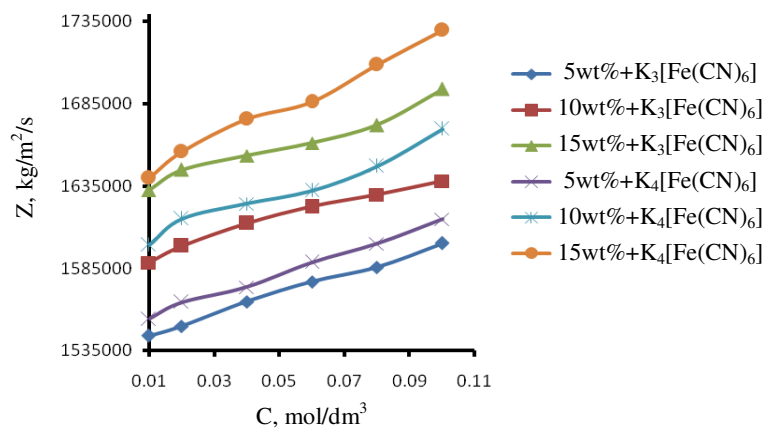

Figure 1. Plot of $Z$ versus $c$ of the solution of potassium ferri and ferro cyanide in different wt $\%$ of dextrose solutions

The increase in $Z$ values with solute concentration can be attributed to the effective solute-solvent interactions. A similar type of behavior has been obtained for various salts studied in different solvent systems. Since the acoustic impendence is a measure of the resistance offered by the liquid medium to the sound wave and is a function of the elastic property of the medium, i.e., the bulk modulus of elasticity, which depends on the structural changes of the solution, the higher $Z$ values of potassium ferrocyanide in the aqueous 
dextrose solutions as than that of ferricyanide and increasingly higher values with increase in the solute concentration and also with increase in dextrose content in water point to the fact that the solution medium in each case starts gaining its elastic property. The lower $Z$ values of ferricyanide solutions than that of solutions of ferrocyanide may be due to its lower molecular mass for which the solutions of potassium ferricyanide start losing the elastic property.

The variation of the molar sound velocity $(R)$ with solute concentrations in different $\mathrm{wt} \%$ of dextrose solutions is shown in Figure 2. As observed, the molar sound velocity increases with increase in wt $\%$ of dextrose solutions for both electrolytes in all the solvents.

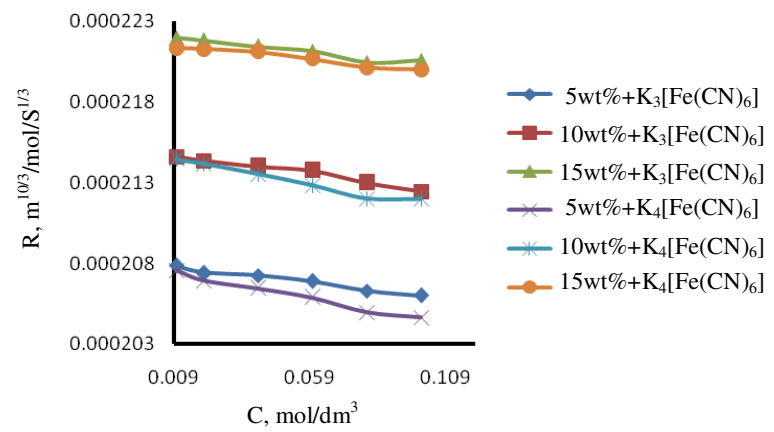

Figure 2. Plot of $R$ versus $c$ of the solutions of potassium ferri and ferrocyanide in different wt $\%$ of dextrose solutions

It is a well known fact that when a solute dissolves in a solvent some of the solvent molecules are attached to the ions (produced from the solute) because of ion-solvent interactions. Since the solvent molecules are oriented in the ionic field (i.e., electrostatic fields of ions) the solvent molecules are more compactly packed in the primary solvation shell as compared to the solvent structure in the absence of the ions. This is the reason why the solvent is compressed by the introduction of ions. Thus, the electrostatic field of the ion causes compression of the medium giving rise to a phenomenon called electrostriction. Since the solvent molecules are compressed they do not respond to any further application of pressure. So the solution becomes harder to compress, i.e., the compressibility decreases and internal pressure increases. Hence, isentropic compressibility as well as internal pressure describe the molecular arrangement in the liquid medium.

The increase in internal pressure, $\pi_{i}$ due to electrostatic field of ions is given by the following equation:

$$
\pi_{i}=\left(K_{s}^{0}-K_{s}\right)
$$

Where $K_{s}^{0}$ and $\mathrm{K}_{s}$ are the isentropic compressibility of the solvent and the solution respectively. Positive values of $\pi_{\mathrm{i}}$ indicate the presence of some specific interactions between unlike molecules in the components. Typical plot of $\pi_{i}$ versus $c$ is shown in Figure 3 for solutions of potassium ferri and ferrocyanide in different $\mathrm{wt} \%$ of dextrose solutions.

Free volume, $V_{f}$, is the effective volume accessible to the centre of a molecule in a liquid. The structure of a liquid is determined by strong repulsive forces in the liquid with the relatively weak attractive forces providing the internal pressure which helds the liquid molecules together. The free volume seems to be conditional by repulsive forces whereas the internal pressure is more sensitive to attractive forces. These two factors together uniquely determine the entropy of the system. Thus, the internal pressure, free volume and temperature seem to be the thermodynamic variables that describe the liquid system of fixed 
composition. It is seen that the free volume, $V_{f}$ increases with solute concentration in all the solvents with slight deviation in $10 \mathrm{wt} \%$ dextrose solution but increases with dextrose content in the mixed solvents. Typical plots are shown in Figure 4.

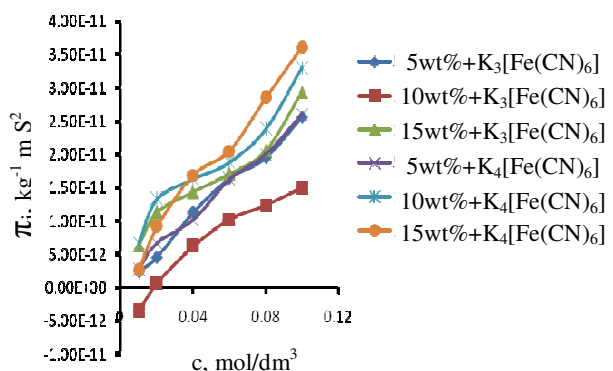

Figure 3. Plot of $\pi_{\mathrm{i}} \quad v s . c$ of the solutions of potassium ferri and ferrocyanide in different wt $\%$ of dextrose solutions

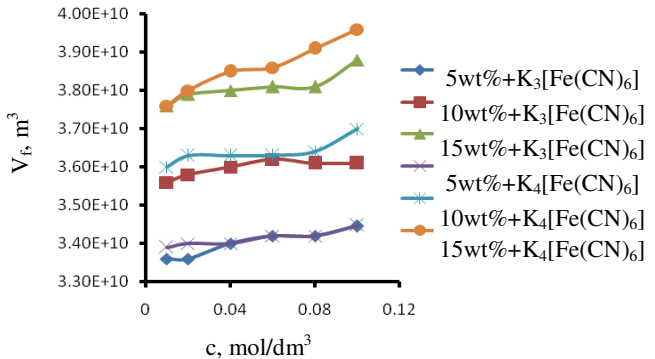

Figure 4. Plot of $V_{f}$ vs. $c$ of the solutions of potassium ferri and ferrocyanide in different $\mathrm{wt} \%$ of dextrose solutions

As observed, the relative association, $R_{A}$ is a function of ultrasonic velocity. The relative association is influenced by two factors: (i) the breaking-up of the solvent structures on addition of solutes to it and (ii) the solvation of solutes. The former results decreases and the latter results increases in $R_{A}$. The increase in $\mathrm{R}_{\mathrm{A}}$ with concentration suggests that the solvation of the electrolytes predominates over the breaking-up of the solvent structure. As observed, $\mathrm{R}_{\mathrm{A}}$ increases exponentially with concentration for both the electrolytes. Typical plots are shown in Figure 5.

Figure 6 shows the variation of molar compressibility $(W)$ for both potassium ferri and ferrocyanide solutions in different wt $\%$ of dextrose solutions. As observed, the values of $W$ increase with increase in the dextrose content in water but decrease with increase in the solute concentrations. The decrease in molar compressibility implies that there is enhanced molecular associations in these systems on increase in solute content, as the new entities formed due to molecular association, become compact and less compressible, satisfying the phenomenon of electrostriction.

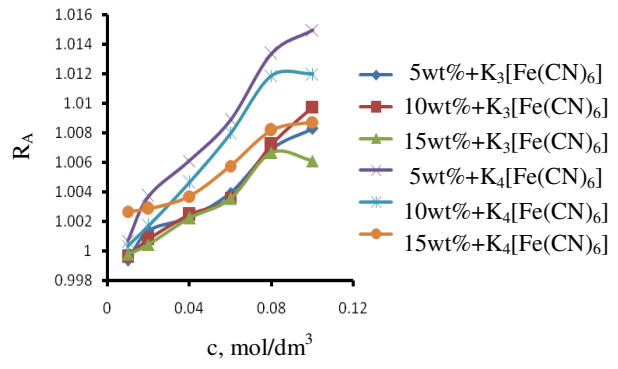

Figure 5. Plot of $\mathrm{R}_{\mathrm{A}}$ versus $c$ of the solutions of potassium ferri and ferrocyanide in different $\mathrm{wt} \%$ of dextrose solutions

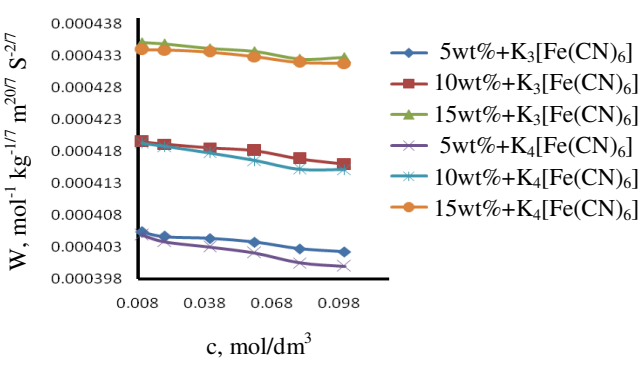

Figure 6. Plot of $W$ versus $c$ of the solutions of potassium ferri and ferrocyanide in different wt $\%$ of dextrose solutions

\section{Conclusion}

From the ultrasonic velocity and density data, various important acoustic parameters like specific acoustic impedance $(Z)$, molar sound velocity $(R)$, molar compressibility $(W)$, free 
volume $\left(V_{f}\right)$, isentropic compressibility $\left(K_{s}\right)$, internal pressure $\left(\pi_{i}\right)$ and relative association $\left(R_{A}\right)$ for the solutions of potassium ferri and ferrocyanide in aqueous dextrose solutions at $298.15 \mathrm{~K}$ have been calculated. The results reveal that the specific ion-ion, ion-solvent and solvent-solvent interactions play an important role for explaining various acoustic parameters. However, any deviation from the usual behavior is probably due to characteristic structural changes in the respective system.

\section{Acknowledgement}

The authors would like to thank Department of Chemistry, ITER, Siksha 'O' Anusandhan University, Bhubaneswar, India for their financial support, and providing the research facilities.

\section{References}

1. Dehury S K and Dash U N, New York Sci J., 2011, 4(11), 64-69.

2. Robinson R A and Stokes R H, Electrolyte Solutions. Butterworths: London; 1955, 30-32.

3. Talukdar M, Moharatha D, Roy G S and Dash U N, Indian J Pure Appl Phys., 2013, 51, 202-206.

4. $\quad$ Das S and Dash U N, J Chem Pharm Res., 2012, 4(1), 754-762.

5. Dash UN, Roy GS, Talukdar M and Moharatha D, Indian J Pure Appl Phys., 2010, 48, 651-657.

6. $\quad$ Dash U N, Mishra S and Samantray B, Egypt J Chem., 2010, 53(1), 163-176.

7. Rama Rao G V, Sandhya Sri P B, Sarma A V and Rambabu C, Indian J Pure Appl Phys., 2007, 45(2), 135-142.

8. Baluja S and Karia F, J Chem Bio Phy Sci., 2011, 2, 101-107.

9. Baskarana R and Kubendran T R, Int J Appl Sci Engg., 2007, 5(2), 115-122.

10. Kavitha R, Jayakumar S and Uma R, Int J Chem Appl., 2011, 3(1), 19-33.

11. Nath G, Chem Sci Trans., 2012, 1(3), 516-521. 